\title{
CALIBRATION RESULTS OBTAINED WITH LIULIN-4 TYPE DOSIMETERS
}

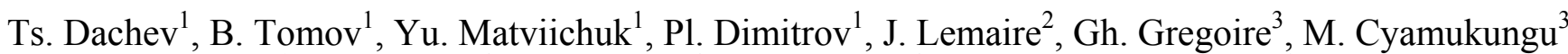

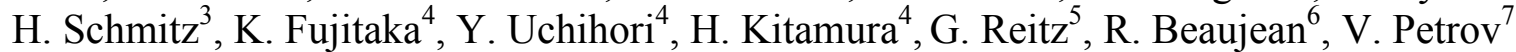 \\ V. Shurshakov ${ }^{7}$, V. Benghin ${ }^{7}$, F. Spurny ${ }^{8}$ \\ ${ }^{1}$ Solar-Terr. Influences Laboratory-BAS, Sofia Bulgaria, tdachev@bas.bg \\ ${ }^{2}$ Institut d'Aeronomie spatiale de Belgique, Bruxelles, j1@oma.be \\ ${ }^{3}$ Institut de Physique, Universite Catholique de Louvain, Belgique, gregoire@fynu.ucl.ac.be \\ ${ }^{4}$ National Institute of Radiological Sciences-STA, Chiba, Japan, fujitaka@,nirs.go.jp \\ ${ }^{5}$ DLR, Institute fuer Luft-und Raumfahrtmedizin, Cologne, Germany, guenther.reitz@dlr.de \\ ${ }^{6}$ Institute fuer Kernphysik des Universitaet Kiel, Germany, r.beaujean@email.uni-kiel.de \\ ${ }^{7}$ Institute of Biomedical Problems, Moscow, Russia, petrov@mmcc.ibmp.rssi.ru \\ ${ }^{8}$ Nuclear Physics Institute, Czech AS, Praha, Czech republic, spurny@ujf.cas.cz
}

\begin{abstract}
The Mobile Radiation Exposure Control System's (Liulin-4 type) main purpose is to monitor simultaneously the doses and fluxes at 4 independent places. It can also be used for personnel dosimetry. The system consists of 4 battery-operated 256-channel dosimeters-spectrometers. We describe results obtained during the calibrations of the spectrometers at the Cyclotron facilities of the University of Louvain, Belgium and of the National Institute of Radiological Sciences-STA, Chiba, Japan with protons of energies up to $70 \mathrm{MeV}$. The angular sensitivities of the devices are studied and compared with MonteCarlo predictions. We also present the results obtained at the HIMAC accelerator with $500 \mathrm{MeV} / \mathrm{u} \mathrm{Fe}$ ions and at the CERN high energy radiation reference fields. Records made during airplane flights are shown and compared with the predictions of the CARI- 6 model.
\end{abstract}

\section{LIULIN-4 SYSTEM DESCRIPTION}

\section{Introduction}

The Mobile Radiation Exposure Control System's (Liulin4) main purpose is to monitor simultaneously the doses and fluxes at four independent places. Liulin-4 precursor was the dosimeter-radiometer Liulin successfully used 1988-1994 on MIR space station Dachev et al., (1989). The system consists of 4 Mobile Dosimetry Units (MDU) and one Control and Interface Unit (CIU) (see Figure 1). The MDU is a miniature spectrometer-dosimeter containing: one semiconductor detector, one chargesensitive preamplifier, 2 microcontrollers; a flash memory and Li-Ion cells. Pulse analysis technique is used for the measurement of the deposited energy in the detector. The unit is managed by the microcontrollers through specially developed firmware. Plug-in links provide the transmission of the stored on the flash memory data through the Control and interface unit toward the standard

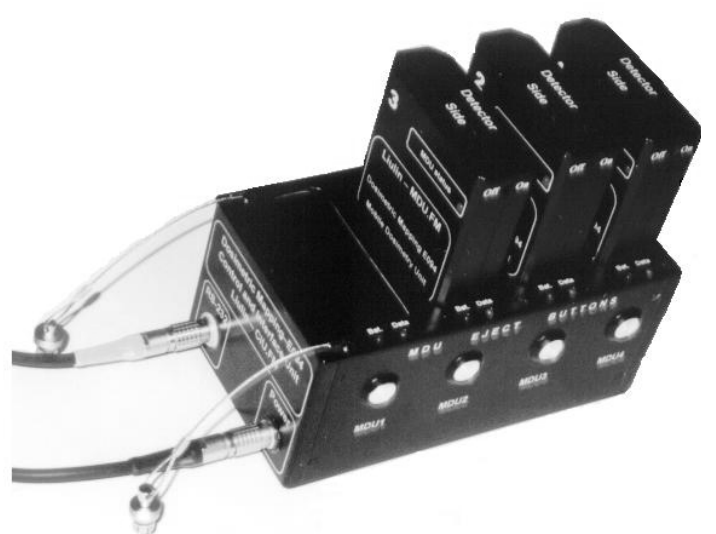

Fig. 1. Picture of the Liulin-E094 Flight model (Only 3 MDUs are inserted into the $\mathrm{CIU})$. 
PC. $28 \mathrm{~V} \mathrm{DC}$ current and DC/DC converter is used for recharging of the Li-Ion sells in the MDUs. CIU is a miniature interface between the PC power supply and the MDUs. Power Supply (external from the system Liulin-4) must generate DC current with voltage between 8.4 and $36 \mathrm{~V}$ and current not less than 0.2 A per MDU. PC provides to CIU standard serial communication port of RS-232 type.

\section{Description of the Mobile Dosimetry Unit (MDU)}

The MDU is designed as a handy spectrometer-dosimeter for continuous monitoring of the radiation environment in different radiation fields. After switching on, the MDU starts to measure in 256 channels the spectra used to calculate the dose and the flux of particles in the silicon detector. The exposition time of one spectra is variable in the interval $10 \mathrm{sec}-3539 \mathrm{sec}$ with 10 -sec step. After finishing the first measurement cycle the spectra, accumulated dose and flux are stored in the flash memory. Each next measurement results are stored in a different place of the memory, which later is used for recalculating of the time of the measurement. After connection of the MDU with the CIU, the MDU transmits to CIU and further to PC all data accumulated.

The MDU contains: one semiconductor detector; one low noise, hybrid, charge-sensitive preamplifier A225 type of AMPTEK inc.; a fast 12 channel ADC; 2 microcontrollers and an flash memory $(0.5$ Mbytes). Pulse high analysis technique is used for measurement of the energy losses in the detector. The unit is managed by 2 microcontrollers through specially developed software. In the MDU a Lithium-Ion battery pack of SONY QN-011BP $7.2 \mathrm{~V}, 1350 \mathrm{mAh}$ type is used. The battery pack consists of 2 cylindrical cells SONY 18650 in 1S-1P configuration. A block schema of portable spectrometerdosimeter in the MDU is presented on Figure 2.

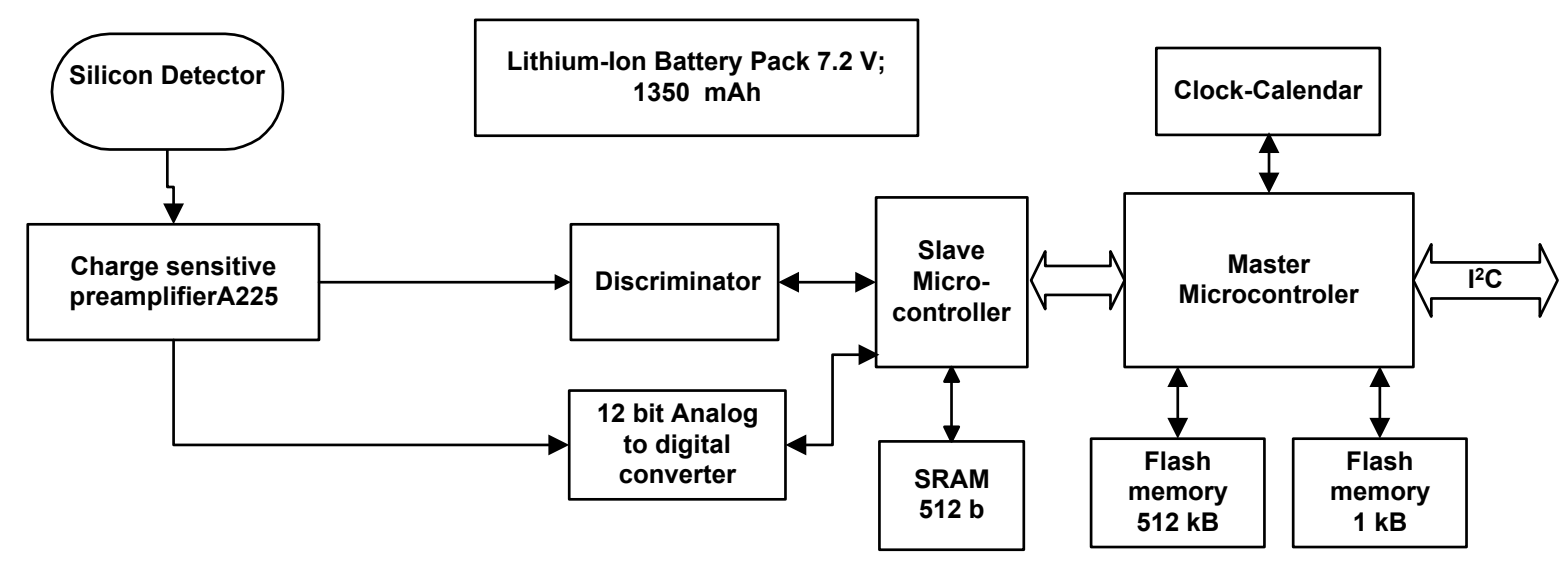

Fig. 2. Block-scheme of the Mobile Dosimetry Unit (MDU).

The main measurement unit in the MDU is the amplitude of the pulses after the preamplifier, which are proportional by a factor of $240 \mathrm{mV} / \mathrm{MeV}$ to the energy loss in the detector and respectively to the absorbed dose. By the 12 bit ADC and by the master processor of the system these amplitudes are digitized and organized in a 256-channel spectra using only the oldest 8 bits of the ADC. The spectra together with information for the real time is saved in the flash memory of the instrument. The capacity of the memory is $0.5 \mathrm{MB}$.

The following method for calculations of the dose is used: The dose D (Gy) by definition is one Joule deposited in $1 \mathrm{~kg}$ or:

$\mathrm{D}=\mathrm{K} \cdot \operatorname{Sum}(\mathrm{Ai} * \mathrm{i}) / \mathrm{MD}$,

where MD is the mass of the detector in $(\mathrm{kg})$. Energy loss in channel $\mathrm{i}$ is proportional to the number of events $A_{i}$ in it multiplied by $i$. Energy loss in the whole detector is a sum of the energy losses in each channel. $\mathrm{K}$ is a coefficient. 
The MDU operate in 3 modes - Working mode, Mode of Data transfer from the flash memory to Control and interface unit and mode of battery recharging:

- In the Working mode the instrument is operating under the software in the microcontrollers. The operational time of the instrument depends on the lifetime of the accumulators and on the rate of the memory fills up. In a case of continuous operation the lifetime is about 120 hours with the standard 1350 $\mathrm{mAh} \mathrm{Li-Ion} \mathrm{accumulators.} \mathrm{The} \mathrm{working} \mathrm{mode} \mathrm{is} \mathrm{switched} \mathrm{OFF} \mathrm{automatically} \mathrm{when} \mathrm{either} \mathrm{the} \mathrm{memory} \mathrm{is}$ totally filled up or the supply voltage is falling below $6.0 \mathrm{~V} \mathrm{DC}$. When the supply voltage of the battery falls down then $6.0 \mathrm{~V}$ the process of measurements stops and the MDU's status indicator start to flash each second, signalizing the necessity for charging of the battery.

- In the mode of data transfer the instrument is switched on by special command when it is connected to one of the 4 sockets of the Control and interface unit after the end of the experiments. The mode allows the transfer of the accumulated in the flash memory data through the CIU to the PC. In this mode the connection of the data with real time and the calculation of the physical values is performed through a special program in the PC.

- The mode of battery recharging is performed when the batteries in MDUs are discharged. The batteries are charged inside of the MDUs with the 4 chargers in the CIU. Charging process is controlled by 4 LT1512 SEPIC Constant Current/Constant voltage battery chargers and is indicated by the red light (Shown with the sign "Bat." on the CIU.). When the red light on CIU is off the battery in the correspond MDU is fully charged. The charging process continues about 15 hours.

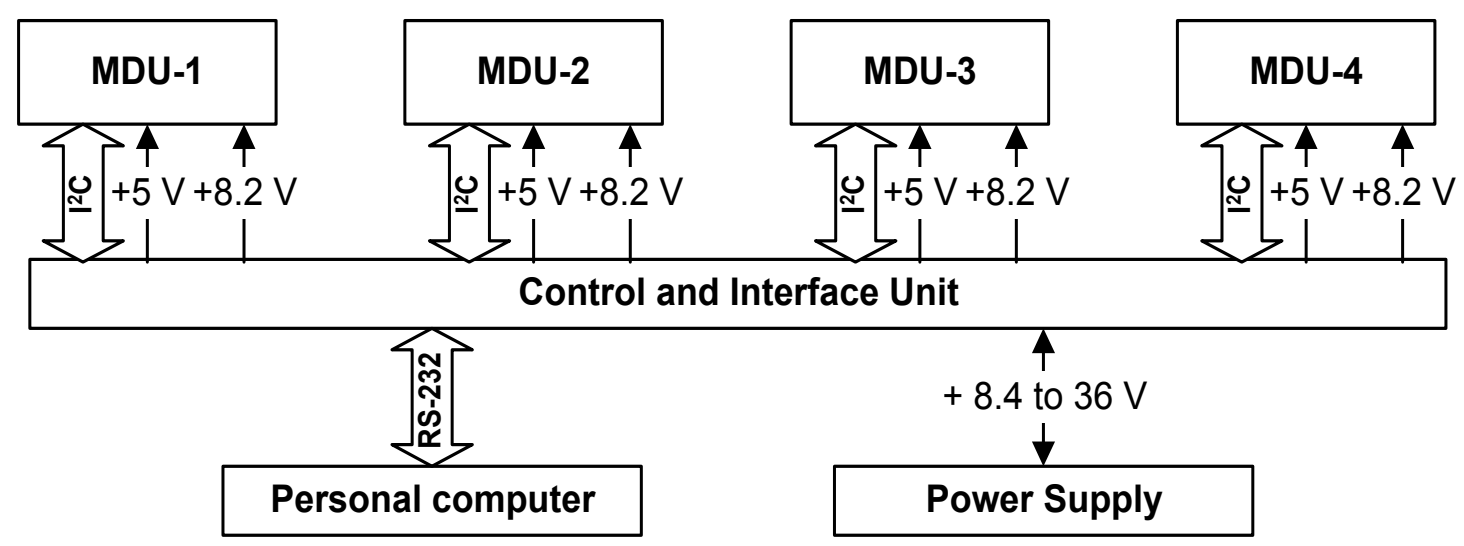

Fig. 3. Place and functions of the Control and Interface Unit.

\section{Description of the Control and Interface Unit}

The Control and Interface Unit (CIU) is a miniature interface between the external power supply, PC and MDUs. Power supply passes to the CIU DC current with the voltage in the interval +8.4 to $36 \mathrm{~V}$ DC (see Figure 3). CIU is connected to PC by standard interface RS-232 type. CIU contains microcontroller,

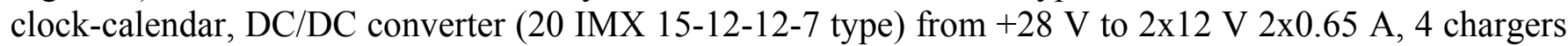
(LT1512 type), one serial communication port of RS-232 type, 4 red LEDs indicating the charging process and 4 yellow LEDs indicating the transfer data process.

The initialization of the Control and Interface Unit is performed automatically when it is connected to the power supply. The success of the internal test is indicated by subsequent turn off of the yellow lamps on CIU. Further CIU continue to work independently till the first connection to the PC. When CIU is connected to PC it takes from the PC system information block the real calendar and clock time. These values are permanently with 10 -sec step compared and updated if necessary with the new real data. Clock-calendar data are transmitted from CIU to each MDUs and used further to evaluate the measurements and to perform them in equal time interval at all 4 MDUs. Simply by default all MDUs 
produces 2 spectra per minute while the first spectra is starting at 00 seconds and the second one at 30 seconds of each minute. Time accuracy of the intervals in the different MDUs is about $10^{-5} \mathrm{~s}$. Data accumulated in the flash memories of MDUs are transmitted through CIU to the PC by the serial interface connection using a specially developed protocol. The Liulin software product (Liulin4X.exe) is developed in "WIN95/NT" environment. At the PC it creates automatically the subdirectory "Liulin-XX Data" in the directory in which "Liulin-4X.exe" is located. In the subdirectory "Liulin-4X Data" two types of files are automatically created. The binary file for each MDU is named automatically and contains in the name YYMMDDHHmm.LIx, where "YYMMDDHHmm" is the date and time of the first measurements with the MDU and " $\mathrm{x}$ " is the number of MDU. This file contains the rough binary data and are for permanent storage of data from the instrument, because of the minimal volume of the file. Two additional ASCII files are created automatically for each binary file. The names of these additional ASCII files contain the same "YYMMDDHHmm" string as the binary files. The files with extensions of type "DIx" and "SIx" contains the "D"ose and "S"pectrum data from each MDU.

\section{LIULIN-4 CALIBRATIONS}

\section{Comparison of Liulin experimental spectra}

Figure 4. presents 7 different experimental spectra, which are obtained with one MDU at different sources and conditions. The lowest in intensity spectrum is obtained at natural background gamma radiation. Here we emphasize the fact that all MDUs do have enough sensitivity to register the natural background gamma radiation. When the value of the measured dose is in the range $0.07-0.14 \mu \mathrm{Gy} /$ hour it is used to check the proper operation of the instrument. Another very low depositing energy spectrum is the $\mathrm{X}$ rays one. The spectrum obtained with ${ }^{137} \mathrm{Cs}$ source is very short because its gamma emission line is centered at $661.7 \mathrm{keV}$. The next relatively short spectra is the ${ }^{90} \mathrm{Sr}-{ }^{90} \mathrm{Y}$ one because the maximum energy of electrons emitted by it is $2.2 \mathrm{MeV}$. The higher energy point is at $1.18 \mathrm{MeV}$, while the higher energy point in the spectrum obtained during an airplane flight at about $12 \mathrm{~km}$ altitude is at $6.14 \mathrm{MeV}$. In other cases at this altitude we observe energy depositions even above the range of the spectrometer, which is $20.7 \mathrm{MeV}$. The maxima of all spectra, except of ${ }^{90} \mathrm{Sr}^{90} \mathrm{Y}$, are situated at $122 \mathrm{keV}$, which is the expected energy loss by relativistic charged particles in 300 microns of silicon. The peak produced by high intensity $60 \mathrm{MeV}$ protons is at 691 $\mathrm{keV}$, while the GEANT code predictions is $710 \mathrm{keV}$. The most right shifted specter maximum is obtained by alpha particles and its maximum energy is $4.35 \mathrm{MeV}$. The

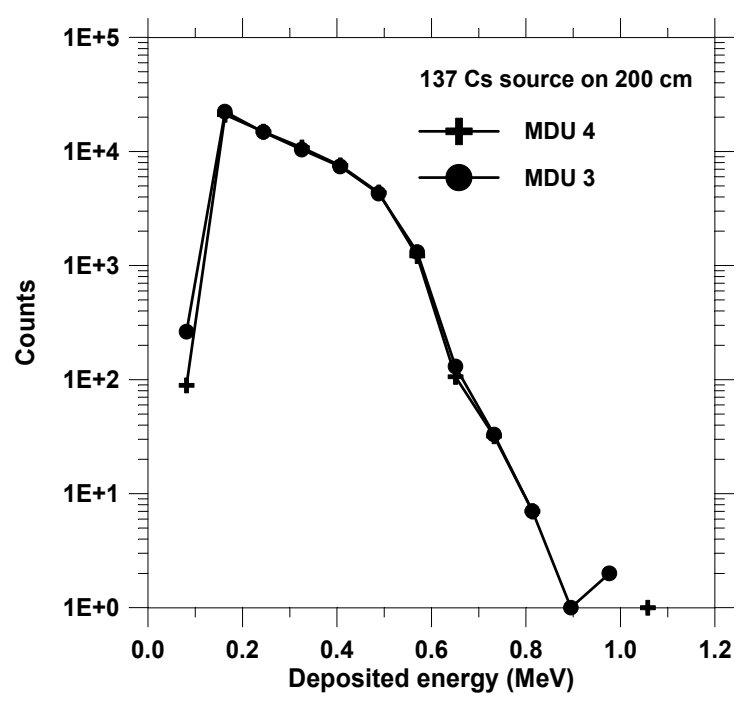

Fig. 5. Spectra obtained with standard ${ }^{137}$ Cs gamma rav source. 
energy reduction from the expected $5.485 \mathrm{MeV}\left({ }^{241} \mathrm{Am}\right.$ alphas) can be explained by the energy degradation in air.

\section{Absolute dosimetric calibrations with standard ${ }^{137}$ Cs source}

Two different MDUs were used to detect the gamma radiation from the source at $2 \mathrm{~m}$ distance. Behind the MDUs there was about 9 meters free space to avoid contamination of the measurements by reflection. One example of the observed mean specters is presented at Figure 5. Two different curves from two different MDUs are seen there, which overlap strongly because the very similar conditions and instrument setup. The absolute predicted at the point of the detectors of the MDUs dose is $3124 \mu \mathrm{Gy} /$ hour in air while the obtained in MDU 3 and MDU 4 doses are 3449.4 and 3459.6 $\mu \mathrm{Gy} /$ hour in Silicon respectively, which gives about $10 \%$ overestimation by Liulin.

\section{Calibrations at the cyclotron in Universite Catholique de Louvain, Louvain-la-Neuve, Belgium}

Our setup uses the W2 area of the Louvain-laNeuve cyclotron. The $65 \mathrm{MeV}$ pencil beam delivered by the accelerator passes first through a $0.25 \mathrm{~mm}$ lead foil to spread it more or less uniformly across the entrance window of the Liulin sensor. This lead foil is located about 4 $\mathrm{m}$ upstream the MDU module. A 30-mm thick brass collimator defines an effective beam spot of about $30 \mathrm{~mm}$ diameter at the sensor position. It is immediately followed downstream the beam line by a thin $(1 \mathrm{~mm})$ scintillation detector NE102 used to check continuously the beam intensity. The MDU is fixed in an aluminum frame mounted on a goniometer. Since the energy losses in the MDU silicon sensor in a case of normal coincidence range between 0.06 (electronic threshold) and $5.7 \mathrm{MeV}$, one chooses incident proton energies so as to sample uniformly the energy deposits between these values. But instead of varying the cyclotron beam energy, we put absorbers of

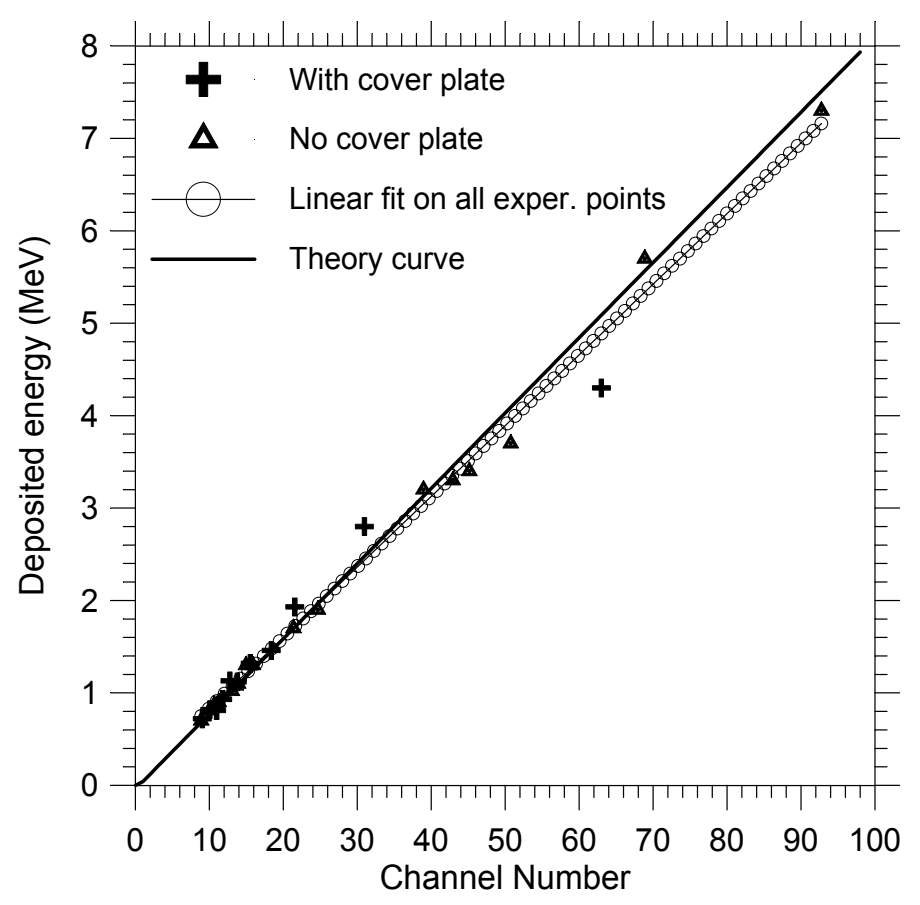

Fig. 6. Centroid positions versus energy losses.

\begin{tabular}{|c|c|c|c|c|}
\hline Absorber & $\begin{array}{c}\text { Thickness } \\
(\mathrm{cm})\end{array}$ & $\begin{array}{l}\text { Expected } \\
\text { energy on the } \\
\text { Liulin Al front } \\
\text { plate }(\mathrm{MeV})\end{array}$ & $\begin{array}{c}\text { Expected } \\
\text { energy loss in } \\
\text { sensor } \\
\text { (unshielded) } \\
(\mathrm{MeV})\end{array}$ & $\begin{array}{c}\text { Expected } \\
\text { energy loss in } \\
\text { sensor } \\
\text { shielded } \\
(\mathrm{MeV})\end{array}$ \\
\hline No & 0.00 & 60.0 & 0.7 & 0.71 \\
Graphite & 1.00 & 41.6 & 0.87 & 0.89 \\
Graphite & 1.40 & 31.9 & 1.02 & 1.05 \\
Graphite & 1.45 & 29.7 & 1.08 & 1.12 \\
Aluminum & 0.4 & 50.5 & 0.79 & 0.81 \\
Aluminum & 1.45 & 7.8 & 3.3 & 3.4 \\
\hline
\end{tabular}

Table 1. Energies expected in front of the Liulin MDU and in the sensor behind different material and thicknesses absorbers. 
known thicknesses in the $65 \mathrm{MeV}$ proton beam delivered by the accelerator. This is much more efficient to change the proton energy at the position of the Liulin MDU. The table 1 lists the various absorber thicknesses chosen for the experiments, the corresponding proton energies at the position of the aluminum front plate and the energy deposits in the Liulin detector. The energy without absorber (first row) is $60 \mathrm{MeV}$ because the incident particles loose about 5 $\mathrm{MeV}$ when crossing the different materials (lead, Mylar, air) along the beam line.

At any given energy and angle of incidence of the protons, a single measurement lasts about 10 seconds. We accumulate several spectra (6 or 12) to increase the statistics. By rotating the MDU around a vertical axis passing in the plane of its entrance face, we vary the angle of incidence $\theta$ of the proton beam between $0^{\circ}$ and $90^{\circ}$.

\section{Analysis of the peak position and calibration curve}

The relation between the channel number and the energy deposition in the Liulin-4 detector is assumed to be linear $\mathrm{E}=\mathrm{aC}+\mathrm{b}$ where $\mathrm{E}$ is the deposited energy in the Silicon sensor and $\mathrm{C}$ is the channel number. The parameters "a" and " $b$ " of the linear fit depend the resolution and accuracy of the ADCs in the MDUs. The predicted formula is found to be: $\mathrm{E}_{\mathrm{t}}=0.081 \mathrm{C}_{\mathrm{t}}$. In the channel number 1 the deposited energy is between 0 and $0.081 \mathrm{MeV}$. The calibrations with $60 \mathrm{keV}$ photons of the ${ }^{241} \mathrm{Am}$ source show us that the first channel has to be divided on 2 parts that is why the formula becomes $\mathrm{E}_{\mathrm{tm}}=0.081 \mathrm{C}_{\mathrm{tm}}-0.0407$ and is shown by the heavy line on Figure 6.

The points shown in Figures 6,7 and 8 were obtained during a calibration runs and also computed using GEANT 3.21 code, Gregoire's programs (Gregoire, (200). Standard peak fitting software and algorithms give the peak positions. The results shown on Figure 6 correspond to the beam test with intensity less than 1000 protons per second across the collimator. Some points of the first experiment were repeated to check the reproducibility. The measurements were first performed without the $0.3 \mathrm{~mm}$ aluminum cover plate on the sensor. Points are shown with $\triangle$. Later we put the aluminum cover plate back to check that the measurements agree with our previous outcomes. These points are shown with + . The linear fit in Figure 6 is obtained with all experimental points and is given by:

$\mathrm{E}=0.0764 \mathrm{C}+0.068$, where $\mathrm{E}$ is the energy deposit in $\mathrm{MeV}$ and $\mathrm{C}$ is the channel number.

Then we evaluate the energy losses using the nominal silicon thickness and tables or software appropriate to low particle energies. The results for the $14.5 \mathrm{~mm}$ aluminum absorbers are displayed in Figure 7., which show the nice

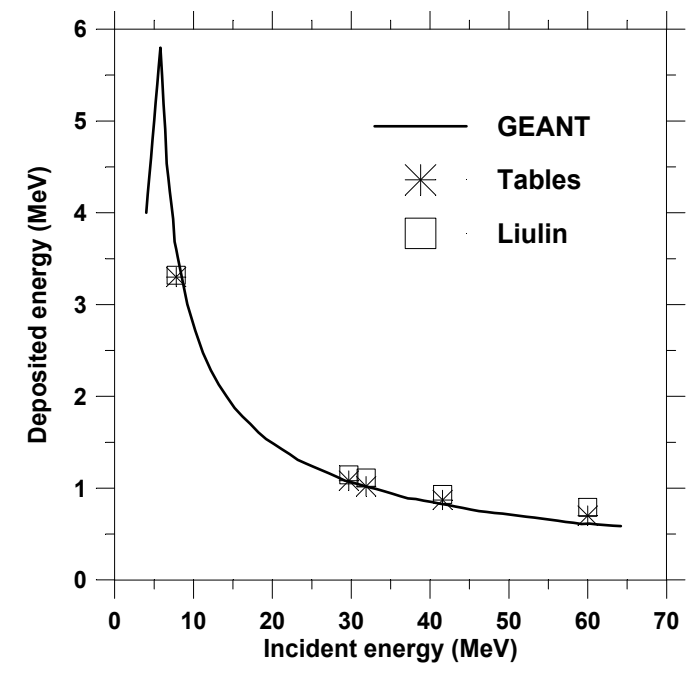

Fig. 8. "Bethe-Bloch" behavior of Liulin experimental data at normal incidence. 
agreements between theoretical and experimental specters at the various incidence angles. The relatively thick aluminum absorber produce large amount of secondary particles including neutrons and the beam at the detector is not pure mono-energetic. Most of these secondaries are not detected and/or do not make any harm to the calibration. The response of the detector against neutrons is presented at Figure 12.

Next we compare the predicted energy losses with the experimental energy deposits at 0 degree incidence angle only. On Figure 8 the "Bethe-Bloch" behavior of Liulin experimental data at normal incidence (pluses) versus energy is shown. The squares refer to Ziegler and Williamson estimations while the continuous line is the prediction of GEANT 3.21.

\section{Calibrations at the cyclotron in National Institute of Radiological Sciences, Chiba, Japan}

Very similar calibrations to the described in the previous paragraph were performed by another set of Liulin MDUs with same mechanical and electronic design at the cyclotron in National Institute of Radiological Sciences, Chiba, Japan. The experimental setup there is simpler than in Louvain-la-Neuve, Belgium. Only $10 \mathrm{~cm}$ air shielding was measured between the cyclotron beam pipe and the Liulin entrance. The obtained results with $40 \mathrm{MeV}$ proton beam is shown on Figure 9 for 4 different incidence angles toward the detector surface $0^{\circ}, 45^{\circ}, 60^{\circ}$ and $75^{\circ}$. In the

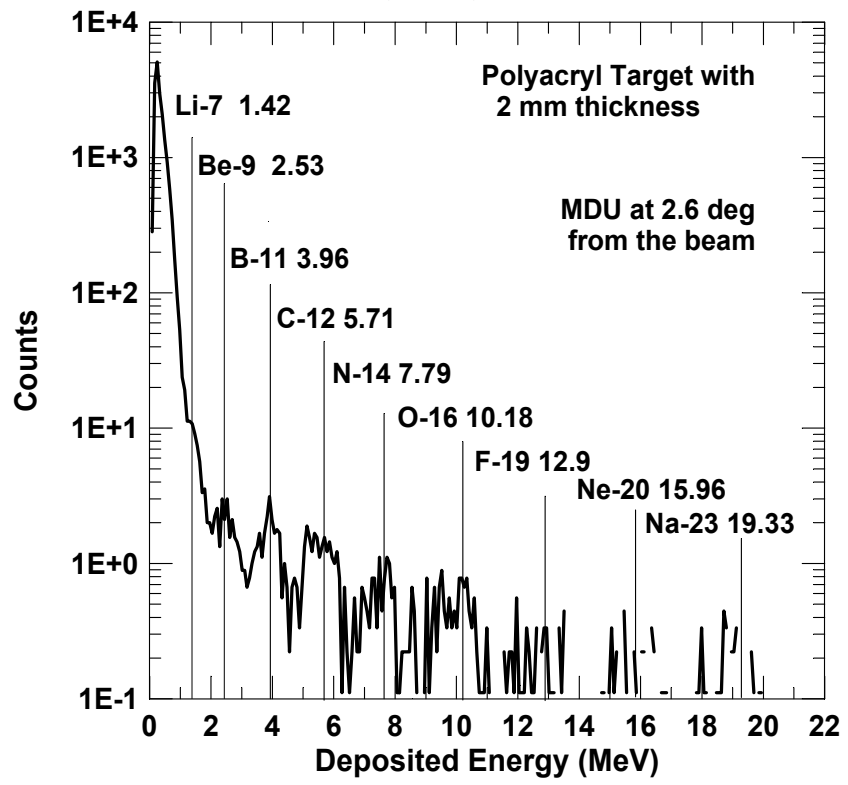

Fig. 11. Example of the fragmentation behind of 2 mm polyacryl target.

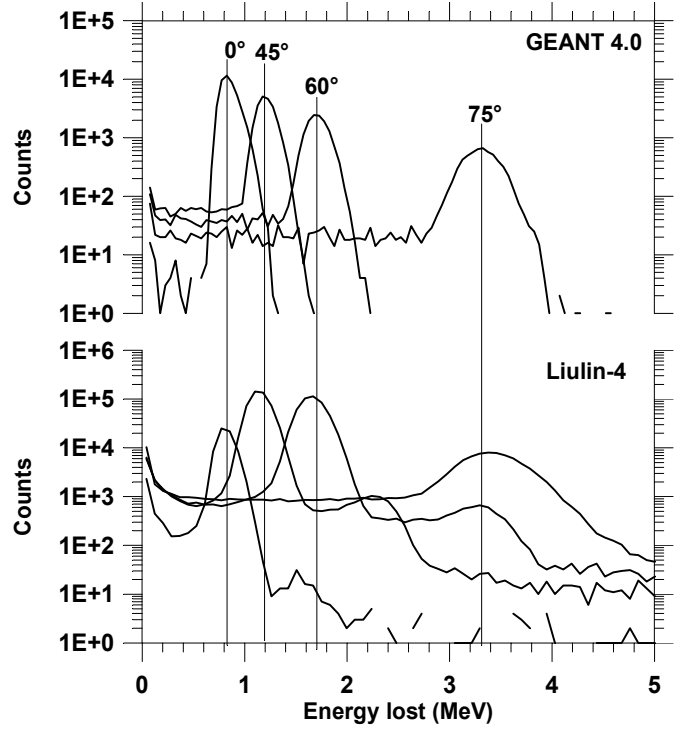

Fig. 9. Liulin and GEANT 4.0 comparisons for $40 \mathrm{MeV}$ proton beam.

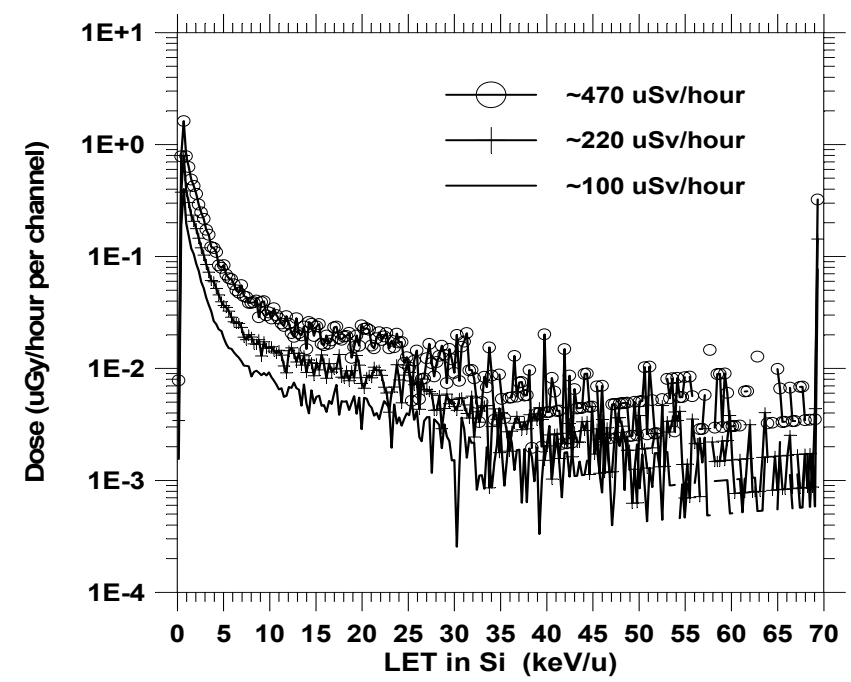

Fig. 10. LET dose distribution in CERN high energy radiation reference field as measured with Liulin dosemeter.

lower panel of the figure the experimental Liulin-4 data are plotted, while the GEANT 4.0 predictions are shown in the upper panel. The dashed vertical lines shows the positions of the predicted by GEANT maximums. It is well seen that the positions of the predicted and measured spectra maximum coincide with accuracy les than $0.2 \mathrm{MeV}$.

\section{Calibrations at the CERN high energy radiation reference field}

Quite recently, we have also tested the behavior of Liulin dosimeter in the high energy radiation reference fields available at CERN and described by 
Höfert, and Stevenson. As far as the CERN high energy radiation reference fields are concerned, we have observed quite good linearity of the response for the beam intensity varying between about 70 and $470 \mu \mathrm{Sv}$ per hour, when expressed in the ambient dose equivalent $\mathrm{H}^{*}(10)$. The response was about 100 pGy (Si) per 1 count of PIC monitor, close to the value corresponding to the low LET radiation contribution. The influence of beam intensity on the spectral distribution observed as also only limited as can be seen in the Figure 10 .

\section{Calibrations at the NIRS-HIMAC heavy ions accelerator}

The experimental setup of the HIMAC was as follow: The $500 \mathrm{MeV} / \mathrm{u}$ Fe ions beam delivered by the HIMAC exit passes first through the target of different thicknesses and materials located at $1 \mathrm{~cm}$ distance from the exit. Next the beam passes of $100 \mathrm{~cm}$ air before the entrance of the MDU sensor. The MDU is fixed on a computer $\mathrm{X}-\mathrm{Y}$ and rotating table. A removable $\mathrm{ZnS}$ plate before the real experiments tests the uniformity of the beam. Plastic scintillation counter was used to measure the count rate of the beam.

When MDU 1 spectrometer was irradiated with iron 500 $\mathrm{MeV} / \mathrm{u}$ ions at $0^{\circ}$ offset from the beam for 30 seconds the major amount of counts are delivered to first 3 channels and in the last channel of the spectra. The deposited energy in the last channel is greater than $20.83 \mathrm{MeV}$ and can be attributed to the primary iron ions. Mainly secondary light ions and protons populate the amount of channels between 255 and 4, while mainly protons and electrons fill the first 3 channels.

On Figure 11 are presented the results obtained by the irradiation of same spectrometer placed at $2.6^{\circ}$ from the main beam for 30 seconds. The spectra presented at Figure 11 is populated by secondary particles, produced by the interaction of the primary $500 \mathrm{MeV} / \mathrm{u}$ iron ions with the polyacryl target with $2 \mathrm{~mm}$ thickness. Lithium, Beryllium Boron, Carbon, Nitrogen, Oxygen, Fluorine, Neon and Sodium ions was recognized in the spectra using the Kanai calculations.

The experiments performed with Liulin-4J instrument on HIMAC facility in our opinion are successful. They show the ability of the instrument to be used for investigation of the fragmentation of the beam after different targets. Calibrations of the instrument with light ions as Neon and Carbon is necessary to be planed in future.

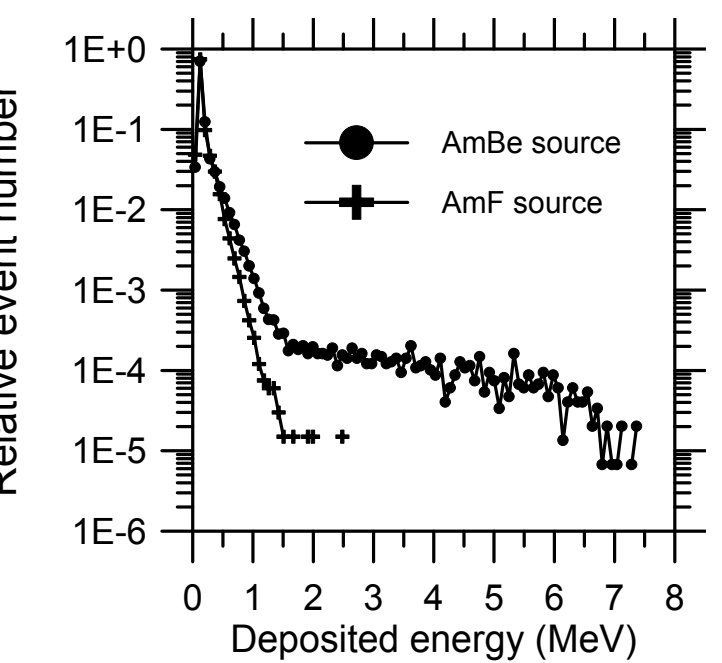

Fig. 12. Experimental spectra obtained by irradiation of Liulin-4C MDU with two different neutron sources.

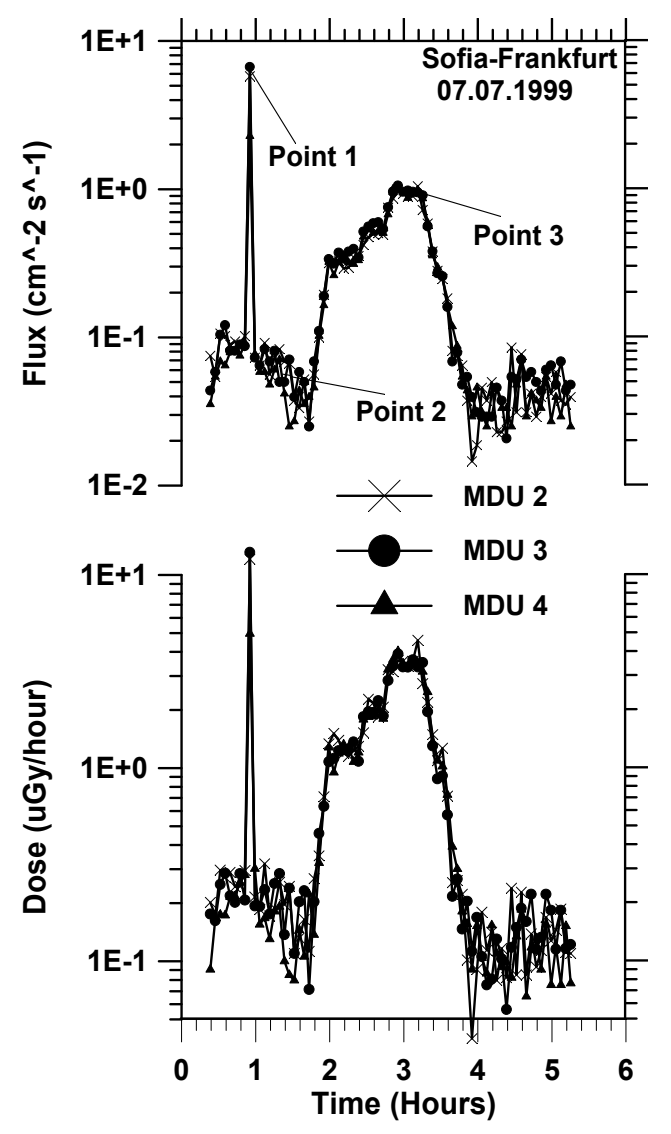

Fig. 13. Flight dose and flux profiles obtained between Sofia and Frankfurt.

\section{Tests with neutron sources}

As far as the neutron exposure is concerned during space experiments we have try to evaluate the Liulin-4 type spectrometers sensitivity toward two different neutron sources. It can be seen from the spectra 
presented in Figure 12 that the energy deposition event are going to much higher energies than in the case of photons (For comparison please see Figure 4.). Of course, the dose absorbed from neutrons in Si calculated from the event spectra is much lower than the kerma in tissue. Relatively it increases from $0.26 \mathrm{MeV}$ for $\mathrm{AmF}$ source with average neutron energy about $1.5 \mathrm{MeV}$ to about 0.60 for $\mathrm{AmBe}$ source with average energy $4.4 \mathrm{MeV}$. What should be stressed is rather large difference in the event spectra characteristic for neutrons as compared to photons. It could permit to distinguish photon and neutron induced events also in other, more complex radiation fields.

\section{AIRCRAFT FLIGHT RESULTS}

On Figure 13 are presented the measured dose and flux profiles from MDU 2, MDU 3 and MDU 4 of the Liulin instrument for the experiment E094 ("Dosimetric mapping") on the American Laboratory module of ISS. Excluding the $\mathrm{X}$-rays security check maximum we see that the measured doses and fluxes in the time before the take off are relatively stable around $0.2 \mu \mathrm{Gy} /$ hour. The most remarkable feature on Figure 11 is the dose and flux decrease seen after the take off of the plane and before the landing. The decrease of the

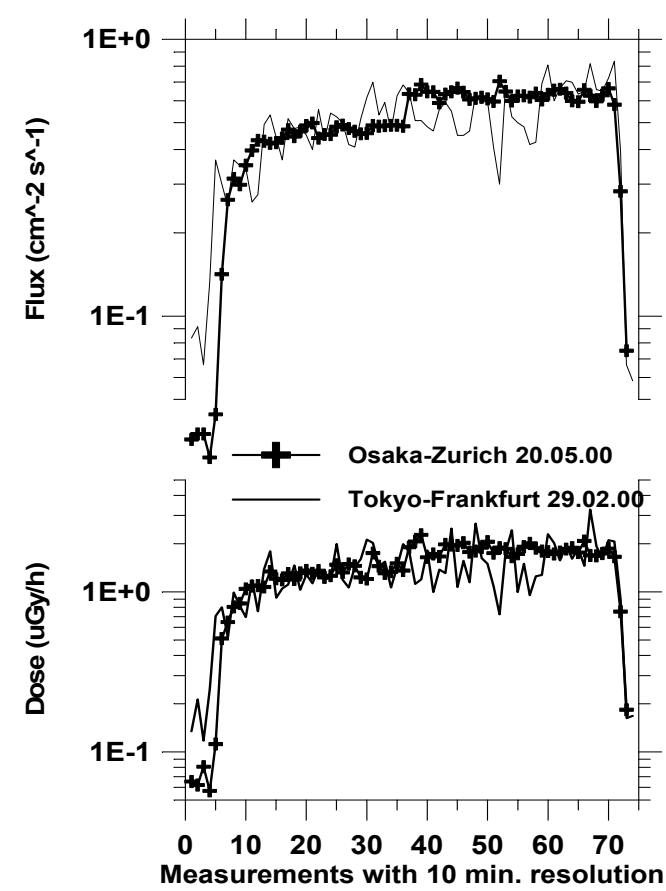

Fig. 14. Latitudinal dependence by graduate increase in the Liulin transcontinental doses and fluxes is seen at left side of the flight profiles. doses and fluxes is because of the air shielding between the ground surface and the airplane. This shielding prevents the penetration in the MDUs the natural background gamma radiation from the earth. When the airplane reaches about $2000 \mathrm{~m}$ the space component of the radiation start to dominate and further with the raising up of the altitude this component increase rapidly. The flight profile with the Russian build Tupolev-154 aircraft has 3 steps in altitude, which are well seen on the dose and flux measurements. "Point 1" mentioned on

the figure correspond to the X-ray security check at the Sofia airport. "Point 1 " mentioned on the figure correspond to the X-ray security check at the Sofia airport. The spectra obtained at this point is seen on Figure 4 and is marked with "x". The spectra in "Point 2" is shown on Figure 4 and marked with "+". The spectra in "Point 3" is marked with "•". For the purposes of statistical analysis the Table below is prepared for the points along the path at which the measured doses exceeds $3 \mu \mathrm{Gy} /$ hour (7-8 points close to the mentioned "Point-3" on the figure). It is well seen from the table that the standard deviations in the measured values of one MDU do not exceeds $14 \%$, which is good result keeping in mind the high probability in the dose measurements. The intercomparison of the MDU 1 with MDU 2 for example show that the dose differences in average dose is $2.61 \%$ and the same calculation of MDU 2 and MDU 4 show $2.63 \%$.

\begin{tabular}{|l|l|l|l|}
\hline Parameter & MDU 2 & MDU 3 & MDU 4 \\
\hline Number of cells & 7 & 7 & 8 \\
\hline Average $(\mu \mathrm{Gy} / \mathrm{h})$ & 3.58 & 3.49 & 3.49 \\
\hline Stand. Deviation $(\mu \mathrm{Gy} / \mathrm{h})$ & 0.476 & 0.212 & 0.268 \\
\hline Standard Deviation $(\%)$ & 13.3 & 6.1 & 7.7 \\
\hline Minimum $(\mu \mathrm{Gy} / \mathrm{h})$ & 3.15 & 3.33 & 3.15 \\
\hline Maximum $(\mu \mathrm{Gy} / \mathrm{h})$ & 4.58 & 3.89 & 4.33 \\
\hline
\end{tabular}

Table 2.

CARI-6 model developed by Friedberg et al. predictions for a point with geographic coordinates 48 North $15^{\circ}$ East and $12000 \mathrm{~m}$ (39390 foots) for July 1999 is $5.96 \mu \mathrm{Sv}$ /hour. This point is close to "Point-3" mentioned on Figure 13. The ratio between CARI-6 prediction and our data is 1.89 for MDU 2 and MDU 4 and 1.79 for MDU 3. 
The latitudinal dependence in the dose and flux distribution is well seen at Figure 14. Two independent sets of measurements were selected from the available number of transcontinental flights between Europe and Japan on 29.02.00 and on 20.05.00. Both flights are on very similar routes from Tokyo and Osaka to Frankfurt and Zurich respectively. On the left hand side part of the profiles on Figure 14 a gradually increase of doses and fluxes is seen, which in our understanding are generated by the gradual increase of the geomagnetic latitude. The "steps" in the right hand side of the profiles are connected with increase of the aircraft altitude at the end of flight.

On Figure 15 is shown two specters obtained on low and high latitudes. Both specters are for 16 minutes of flight and are presented in a coordinate system Dose Rate (uGy/hour) versus Linear energy transfer in Silicon. The first remarkable feature of the picture is that the increase of the latitude increases totally the whole spectra. Falling part of the specters is under a power low organization and the power low formulas for the both specters are shown in the bottom of the figure. The major part (94\%) of the doses is delivered by particles with LET below 10 $\mathrm{keV} / \mathrm{u}$. In the maximum the deposited doses reaches $2 \mu \mathrm{Gy} /$ hour. The part of specters above $10 \mathrm{keV} / \mathrm{u}$ is produced by single events and the rising doses in it are attributed to the rising number of the channel, from which the dose values depends.

\section{CONCLUSDIONS}

The results of our studies have shown, we believe, that the Liulin-4 type dosimeter with MDU units represent a very useful, versatile and flexible facility to monitor the absorbed dose from many types of ionizing radiation and the charged particle fluence rates.

During the absolute dosimetric calibrations at the ${ }^{137} \mathrm{Cs}$ facility was found that Liulin dosimeters overestimated the predicted values by $10 \%$. This result can be easy understand having in mind the fact that movement of the specter by 1 step in left or right direction bring $\pm 40 \%$ differences in the dose.

As far as the absorbed dose rate is concerned it was shown that the dosimeter could reasonably correctly estimate the low LET radiation doses within the range of dose rates from the natural background $(\sim 100$ $\mathrm{nGy} / \mathrm{h}$ ) up to, at least, few $\mathrm{mSv} / \mathrm{h}$. The minimum observed doses in a high-shielded environment by MDU is $44 \mathrm{nGy} /$ hour. Some additional studies seem to be useful to verify the instrument's calibration using more large scale of particle types and theirs energies.

As far as the charged particle fluence rate is concerned, the instrument permits to register particles with fluence rates between 0.01 and $1000 \mathrm{~cm}^{-2} \mathrm{~s}$. Spectrometry quality of the instrument permit to determine also the quality of radiation, i.e. the particle type and its energy in a case of simple radiation field. In more complicated radiation field the recognition of the primary radiation sources.

The calibrations in the Louvain-la-Neuve cyclotron facility shows that the predicted proton energy losses computed by Ziegler and Williamson's tables agree fairly well with the measurements. The small differences between experimental and predicted data may come from inaccuracy on the experimental incidence angles read on the goniometer. GEANT correctly predicts the energy losses above $8 \mathrm{MeV}$. In this range, the observed angular dependence of the energy losses is in good agreement with GEANT 
predictions. At lower than $8 \mathrm{MeV}$ incident energies, the GEANT 3.21 code is inappropriate to predict the energy losses.

The experiments performed with Liulin-4J instrument on HIMAC facility show the ability of the instrument to be used for investigation of the fragmentation of the beam after different targets. Calibrations of the instrument with light ions as Neon and Carbon is necessary to be planed in future.

The large difference in the event spectra characteristic for neutrons as compared to photons could permit to distinguish photon and neutron induced events. This study should continue in the future.

The obtained aircraft results are in good agreement with the CARI-6 model predictions and demonstrate the ability of the instrument to be used for dose and flux measurements in aircraft and spacecraft measurements and especially on the International Space Station. Further use of Liulin-4 dosimeters in aircraft and balloons experiments may give additional data for intercomparison of real and predicted by Cary-6 model latitudinal profiles.

\section{ACKNOWLEDGEMENTS}

This work is partly supported by: 1) Agreement between STIL-BAS and DLR and Kiel University, Germany; 2) Liulin-4 Agreement, leaded by SSTC, number IN/BW/004 between STIL-BAS and the Belgian Institute for Space Aeronomy, Brussels and Institut Georges Lemaitre and the Institut de Physique Nucléaire of the Université Catholique de Louvain, Belgium; 3) Memorandum of Agreement between STIL-BAS and the National Institute for Radiological Sciences, Chiba, Japan; 4) CONTRACT No NZ-701 of 24.11.1997 between STIL-BAS and the National scientific fund of Bulgaria. Bulgarian authors are thankful to Dr. Zdravko Buchakliev, which perform the absolute calibrations with ${ }^{137} \mathrm{Cs}$ source.

\section{REFERENCES}

Dachev, Ts. P., Yu. N. Matviichuk, J. V. Semkova, R. T. Koleva, B. Boichev, P. Baynov, N. A. Kanchev, P. Lakov, Ya. J. Ivanov, B.T. Tomov, V. M. Petrov, V. I. Redko, V. I. Kojarinov, R. Tykva, Space radiation dosimetry with active detection's for the scientific program of the second Bulgarian cosmonaut on board the MIR space station, Adv. Space Res., 9, 10, 247-251, 1989.

Friedberg W., K. O'Brien, III, D.E. Parker, M.A. Shea, D.F. Smart, CARI-6 model, http://www.cami.jccbi.gov/aam-600/610/600radio.html\#CARI6EXE.

GEANT Version 3.21, Simulation package, CERN, Geneva.

Gregoire, Gh., VRANGE and VSTOP range/stopping power programs based on Ziegler's tables, Williamson's tables and Hubert's tables, private communication, 2000.

Höfert, M., G.R. Stevenson: "The CERN-CEC High Energy Reference Field Facility". Contribution presented at the American Nuclear Society Meeting - 8th Inter. Conf. on Radiation Shielding, Arlington, Texas, April 1994.

Hubert et al., Annales de Physique, S-5, 1980.

Kanai T., private communication, 1998.

Peakfit Version 4.0, Peak separation and analysis software, Jandel Scientific software, 1982.

Williamson, C. et al., Saclay report CEA-R3042, 1966.

Ziegler, J.F., H.H. Anderson, The stopping power and range of ions in all elements, New York, Pergamon Press, 1977. 